\title{
Evaluation of biotherapies T.cruzi 15x, 16x, 17x and "potency chords" in experimental infection by Trypanosoma cruzi.
}

\author{
Fabiana Nabarro Ferraz, Valdir Antonio Gonçalves, Denise Lessa Aleixo, \\ Angélica Sayuri Mizutani, Silvana Marques de Araújo
}

State University of Maringá, Maringá, PR, Brazil

\begin{abstract}
Background: The biotherapies are drugs widely utilized against infectious diseases. Biotherapies' profylatic and therapeutic action against Chagas Disease is currently being investigated, but it is needed to develop further controlled experiments "in vivo", which could define more clearly: dilution, dose, time of use and, if possible, the action mechanisms of these ultradiluted medicaments [1,2].
\end{abstract}

Aim: Evaluate the effect biotherapies T. cruzi 15x, 16x, 17x and "potency chords", on experimental infection by T. cruzi.

Methodology: A blind, controlled and randomized by drawing test was performed. Animals: 29 male Swiss mice, four weeks old were utilized. The animals were kept at Parasitology Vivarium/State University of Maringá (UEM), in ideal conditions of temperature $(22 \pm 2)^{\circ} \mathrm{C}$ and photoperiod (light / dark cycle 12h). Mice have been inoculated intraperitoneally with 1400 blood trypomastigotes Y strain and divided in groups: IC Infection control (treated with distilled water - 9 animals); TBBA $15 x 3$ days - Treated with biotherapy $15 \mathrm{x} 3$ days before and 3 days after infection ( 5 animals); TBBA $16 x 3$ days - Treated with biotherapy 16x 3 days before and 3 days after infection ( 5 animals); TBBA $17 x 3$ days - Treated with biotherapy $17 \mathrm{x} 3$ days before and 3 days after infection ( 5 animals); TBBAchords 3 days - Treated with biotherapy 15x, 16x, 17x "potency chords", 3 days before and 3 days after infection (5 animals).

Biotherapies: prepared by a homeopathic pharmacist from UEM, according to Farmacopéia Homeopática Brasileira [3].

Biotherapies treatment schedule: diluted in distilled water $(10 \mu \mathrm{L} / \mathrm{mL}$ in ambar bottles - renewed each two days) offered ad libitum, 3 days before and 3 days after infection in all groups.

Parasitological parameters: parasitemia was assessed from infection until death, according to Brener's technique [4] with $5 \mu \mathrm{L}$ of blood collected from the tail vein and examined in optical microscope. Pre-patent period, patent period, total parasitemia, survival and morbidity were obtained from the parasitemia curve.

Clinical parameters: Visually assessed (presence or absence): body hair aspect (bristling), edema, movement and diarrhea. Measured: body weight, temperature, food and water intake [5].

Ethics: This study has been approved by the UEM Ethics Committee for Experiments in Animals Registration 030/2008. Statistical analysis: was performed using the tests Kruskal Wallis and Mann-Whitney tests, significance of $5 \%$. 
Results: There was not statistical difference between total parasitemia of the groups treated with biotherapies and the IC group ( $\mathrm{p}=0.6819$ ). The parasitemia curve of group TBBAChords3days was greater then the IC $(\mathrm{p}=0.0418)$. Despite this increase, patent period and mortality both showed a decreasing tendency, while pre patent period and survival time increased $(\mathrm{p}=0.373)$. The same tendency results were observed for TBBA $_{17 \times} 3$ days results (Table 1 ). Survival of at least one mice in groups TBBA $_{17 \times} 3$ days and TBBAchords 3 days is worthy of discussion, since Y strain causes $100 \%$ mortality in these experimental conditions.

Table 1. Parasitological parameters (mean \pm standar deviation) evaluated in Swiss male mice experimentally infected by T. cruzi, submitted to treatment with biotherapies 15x, 16x, 17x and "Potency Chords".

\begin{tabular}{lccccc}
\hline Group & $\begin{array}{c}\text { Pre patent } \\
\text { period (days) }\end{array}$ & $\begin{array}{c}\text { Patent Period } \\
\text { (days) }\end{array}$ & $\begin{array}{c}\text { Total parasitemia } \\
\text { (trypomastigotes/mL) }\end{array}$ & $\begin{array}{c}\text { Survival } \\
\text { (days) }\end{array}$ & $\begin{array}{c}\text { Mortality } \\
\text { (\%) }\end{array}$ \\
\hline IC & $6.8 \pm 3.0$ & $7.8 \pm 2.1$ & $(81 \pm 21) \times 10^{4}$ & $16.3 \pm 2.5$ & 100 \\
TBBA $15 x 3$ days & $6.0 \pm 2.9$ & $8.8 \pm 1.3$ & $(83 \pm 32) \times 10^{4}$ & $16.0 \pm 2.8$ & 100 \\
TBBA $16 x 3$ 3ays & $5.0 \pm 1.7$ & $8.6 \pm 1.1$ & $(114 \pm 59) \times 10^{4}$ & $15.8 \pm 2.9$ & 100 \\
TBBA $17 x 3$ days $_{\text {TBBAAcorde3days }}$ & $7.6 \pm 8.0$ & $6.8 \pm 3.8$ & $(70 \pm 49) \times 10^{4}$ & $52 \pm 72$ & 80 \\
\hline
\end{tabular}

Groups TBBA $_{17 \times} 3$ days and TBBAChords 3 days showed better evolution than IC group for body weight, temperature, food and water intake $(\mathrm{p}=0.05)$, body hair aspect and edema developing. Diarrhea and hind legs paralysis were only observed in mice belonging to groups IC and TBAA $16 x 3$ days.

Conclusions: Superior effect was obtained with biotherapies 17x and "Potency Chords", both for clinical and parasitological parameters. "Potency chords" has proper effect which distinguishes it from the individual effects of the dilutions that compound it.

Key-words: Trypanosoma cruzi; Chagas Disease; Biotherapies, "Potency chords".

\section{Refererences}

[1] Almeida LR. Pesquisa em Bioterápicos. Cultura Homeopática. 2006; 16:12.

[2] Ferraz FN, Sandri P, Aleixo DL, Spack M, Júnior ADN, Gomes ML, Araújo SM. O efeito de diferentes potências de bioterápico de Trypanosoma cruzi na infecção experimental. In: VII Congresso Brasileiro de Farmácia Homeopática, 2009, Águas de Lindóia. Brazilian Homeopathic Journal. 2009; 11:5-6.

[3] Farmacopéia Homeopática Brasileira: Parte I - Métodos Gerais, 2ed, São Paulo: Atheneu; 1997.

[4]. Brener Z. Therapeutic activity and criterion of cure on mice experimentally infected with Trypanosoma cruzi. Rev Inst Med Trop São Paulo. 1962; 4: 389-96.

[5] Falkowski GJS , Tiyo R, Aleixo DL, Sandri PF, Araújo SM. Parameters for evaluation of clinical trial in mice. Submitted to the Arquivo Brasileiro de Medicina Veterinaria e Zootecnia - registered as ID 4508/2011. 


\section{Avaliação do efeito de bioterápicos 15x,16x, 17x e acorde de potências de $T$.cruzi sobre a infeção experimental pelo Trypanosoma cruzi.}

\section{RESUMO}

Introdução: Os bioterápicos são medicamentos amplamente utilizados contra doenças infecciosas. A ação profilática e terapêutica dos bioterápicos contra a doença de Chagas está atualmente sendo investigada, havendo ainda a necessidade do desenvolvimento de novos experimentos controlados "in vivo", que definam mais claramente: diluição, dose, tempo de utilização e se possível mecanismos de ação destes ultradiluídos $[1,2]$.

Objetivo: Avaliar o efeito de bioterápicos de T. cruzi 15x, 16x, 17x e Acorde destas potências, na infecção experimental pelo T. cruzi.

Metodologia: Foi realizado um experimento cego, controlado e randomizado por sorteio. Animais: Foram utilizados 29 camundongos suíços, machos com quatro semanas de idade. Os animais foram mantidos em biotério, na Universidade Estadual de Maringá (UEM), em condições ideais de temperatura $\left(22^{\circ} \mathrm{C} \pm 2^{\circ} \mathrm{C}\right)$ e fotoperíodo (ciclo claro/escuro 12h). Os camundongos foram inoculados com 1400 tripomastigotas sanguíneos da cepa Y, via intraperitonial e divididos nos seguintes grupos: CI - Controle de infecção (tratados com água destilada - 9 animais); TBAD $15 x 3$ dias - Tratados com bioterápico $15 \times 3$ dias antes e 3 dias depois da infecção (5 animais); TBAD ${ }_{16 x} 3$ dias - Tratados com bioterápico 16x 3 dias antes e 3 dias depois da infecção (5 animais); TBAD $_{17 x} 3$ dias - Tratados com bioterápico $17 \times 3$ dias antes e 3 dias depois da infecção (5 animais);

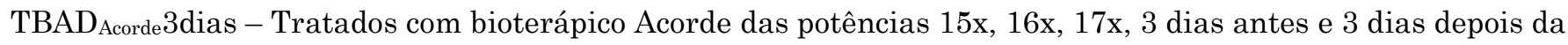
infecção (5 animais).

Bioterápicos: Foram preparados segundo a Farmacopéia Homeopática Brasileira, por um farmacêutico homeopata da UEM [3].

Esquema de utilização dos bioterápicos: os ultadiluídos foram oferecidos ad libitum, em água destilada $(10 \mu \mathrm{L} / \mathrm{mL}$ em bebedouro âmbar - renovada a cada dois dias), 3 dias antes e 3 dias depois da infecção em todos os grupos.

Parâmetros Parasitológicos: a parasitemia foi avaliada utilizando a técnica de Brener [4] com 5 $\mu \mathrm{L}$ de sangue retirado da veia caudal e examinado entre lâmina e lamínula no microscópio óptico. A partir da curva de parasitemia foram obtidos: período pré-patente, patente, parasitemia total, sobrevida e morbidade.

Parâmetros clínicos: Foram avaliados visualmente (presente ou ausente): aspecto da pelagem (eriçamento), edema, movimento e diarréia. Foram mensurados: peso, temperatura, consumo de ração e água [5].

Ética: Este trabalho foi aprovado pelo Comitê de Ética em Experimentação Animal da Universidade Estadual de Maringá - Parecer nº 030/2008.

Análise estatística: Foram realizados os testes de Kruskal Wallis e Mann-Whitney, nível de significância de $5 \%$.

Resultados: Não houve diferença estatística entre a parasitemia total dos grupos tratados com bioterápicos e o grupo CI $(\mathrm{p}=0,6819)$. A curva de parasitemia do grupo $\mathrm{TBAD}_{\text {Acorde }} 3$ dias foi maior que a do CI ( $\left.\mathrm{p}=0,0418\right)$. Apesar deste aumento, o período patente e mortalidade mostraram tendência a diminuir, enquanto o período 
pré-patente e sobrevivência aumentaram $(\mathrm{p}=0,373)$. Resultados com a mesma tendência foram observados para o grupo TBAD ${ }_{17 x} 3$ dias (Tabela 1). A sobrevivência de pelo menos um camundongo nos grupos $\mathrm{TBAD}_{17 \times} 3$ dias e $\mathrm{TBAD}_{\text {Acorde}} 3$ dias, é digno de discussão, visto que a cepa Y provoca invariavelmente $100 \%$ de mortalidade nesta condições experimentais.

Table 1. Parâmetros parasitológicos (média \pm desvio padrão) avaliados em grupos de camundongos suíços, machos, experimentalmente infectados com $T$. cruzi, submetidos ao tratamento com bioterápico de $T$. cruzi 15x, 16x, 17x e Acorde de potências.

\begin{tabular}{lccccc}
\hline Grupo & $\begin{array}{c}\text { Periodo pré } \\
\text { patente (dias) }\end{array}$ & $\begin{array}{c}\text { Periodo } \\
\text { Patente (dias) }\end{array}$ & $\begin{array}{c}\text { Parasitemia total } \\
\text { (tripomastigotas/ml) }\end{array}$ & $\begin{array}{c}\text { Sobrevida } \\
\text { (dias) }\end{array}$ & $\begin{array}{c}\text { Mortalidade } \\
\text { (\%) }\end{array}$ \\
\hline IC & $6,8 \pm 3,0$ & $7,8 \pm 2,1$ & $(81 \pm 21) \times 10^{4}$ & $16.3 \pm 2,5$ & 100 \\
TBBA $_{15 x} 3$ dias & $6,0 \pm 2,9$ & $8,8 \pm 1,3$ & $(83 \pm 32) \times 10^{4}$ & $16.0 \pm 2,8$ & 100 \\
TBBA $_{16 \times} 3$ dias & $5,0 \pm 1,7$ & $8,6 \pm 1,1$ & $(114 \pm 59) \times 10^{4}$ & $15.8 \pm 2,9$ & 100 \\
TBBA $_{17 \times 3 d i a s}$ & $7,6 \pm 8,0$ & $6,8 \pm 3,8$ & $(70 \pm 49) \times 10^{4}$ & $52 \pm 72$ & 80 \\
TBBA Acorde3dias & $10,2 \pm 6,8$ & $5,8 \pm 3,4$ & $(115 \pm 86) \times 10^{4}$ & $52 \pm 41$ & 80 \\
\hline
\end{tabular}

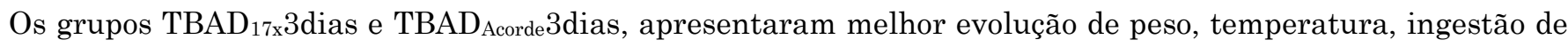
ração e água $(\mathrm{p}=0,05)$ que o $\mathrm{CI}$, isso também foi visualizado em relação ao aspecto dos pêlos e desenvolvimento de edema . A diarréia e a paralisia de patas traseiras só foram observadas em camundongos pertencentes ao grupo CI e TBAD $16 x 3$ dias.

Conclusão: $\mathrm{O}$ melhor efeito foi obtido com bioterápico 17x e Acorde de potências, para parâmetros clínicos e parasitológicos. O Acorde de potências apresenta efeito próprio que o diferencia dos efeitos individuais das diluições que o compõem.

\section{(c) EY-NC-ND Licensed to GIRI}

Support: Fundação Araucária. The authors had full access to all the data in this study and they take complete responsibility for the integrity of the data and the accuracy of the data analysis.

Conflict of interest: authors declare there is no conflict of interest

Correspondence author: Fabiana Nabarro Ferraz, fabiana_nabarro@hotmail.com .

How to cite this article: Ferraz FN, Gonçalves VA, Aleixo DA, Mizutani AS, de Araújo SM. Evaluation of biotherapies $t$. Cruzi 15x, 16x, 17x and "potency chords" in experimental infection by Trypanosoma cruzi. Int J High Dilution Res [online]. 2011 [cited YYYY Month dd]; 10(36): 130-133. Proceedings of the XXV GIRI Symposium and VIII CBFH; 2011 Sep 04-07; Foz do Iguaçu (Brazil). GIRI and ABFH; 2011; Available from: http://www.feg.unesp.br/ ojs/index.php/ijhdr/article/view/477/487 